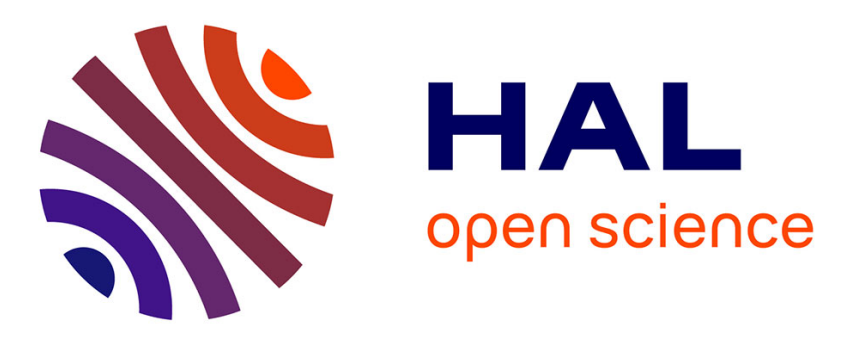

\title{
Normalized Coherency Matrix Estimation Under the SIRV Model. Alpine Glacier Polsar Data Analysis
}

Gabriel Vasile, Jean-Philippe Ovarlez, Frédéric Pascal, Céline Tison, Lionel Bombrun, Michel Gay, Emmanuel Trouvé

\section{- To cite this version:}

Gabriel Vasile, Jean-Philippe Ovarlez, Frédéric Pascal, Céline Tison, Lionel Bombrun, et al.. Normalized Coherency Matrix Estimation Under the SIRV Model. Alpine Glacier Polsar Data Analysis. IGARSS 2008 - IEEE International Geoscience and Remote Sensing Symposium, Jul 2008, Boston, MA, United States. 10.1109/IGARSS.2008.4778796 . hal-00353606

\section{HAL Id: hal-00353606 \\ https://hal-centralesupelec.archives-ouvertes.fr/hal-00353606}

Submitted on 8 Jun 2021

HAL is a multi-disciplinary open access archive for the deposit and dissemination of scientific research documents, whether they are published or not. The documents may come from teaching and research institutions in France or abroad, or from public or private research centers.
L'archive ouverte pluridisciplinaire HAL, est destinée au dépôt et à la diffusion de documents scientifiques de niveau recherche, publiés ou non, émanant des établissements d'enseignement et de recherche français ou étrangers, des laboratoires publics ou privés. 


\title{
NORMALIZED COHERENCY MATRIX ESTIMATION UNDER THE SIRV MODEL. ALPINE GLACIER POLSAR DATA ANALYSIS
}

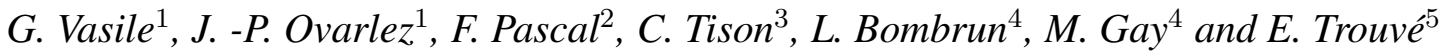 \\ ${ }^{1}$ : ONERA DEMR/TSI, gvasile@onera.fr, ovarlez@onera.fr \\ 2 : SONDRA, frederic.pascal@supelec.fr \\ 3 : CNES DCT/SI/AR, celine.tison@cnes.fr \\ ${ }^{4}$ : GIPSA-lab, CNRS, lionel.bombrun@gipsa-lab.inpg.fr, michel.gay@gipsa-lab.inpg.fr \\ ${ }^{5}$ : LISTIC, Université de Savoie, trouve@univ-savoie.fr
}

\begin{abstract}
This paper presents an application of the recent advances in the field of Spherically Invariant Random Vectors modelling. We propose the use of the Fixed Point (FP) estimator for deriving normalized polarimetric coherency matrices in compound Gaussian clutter. The main advantages of the FP estimator are that it does not require any "a priori" information about the probability density function of the texture and it can be directly applied on adaptive neighborhoods. Interesting results are obtained when coupling this FP estimator with an adaptive spatial support driven on the scalar span information. The proposed method is tested with both simulated POLSAR data and high resolution POLSAR data acquired over the French Alps.
\end{abstract}

Index Terms - polarimetry, SAR, coherence, estimation, SIRV, glaciers

\section{INTRODUCTION}

A Synthetic Aperture Radar (SAR) measures both amplitude and phase of the backscattered signal, producing one complex image for each recording. The sensors being able to emit and receive two orthogonal polarizations, fully POLarimetric Synthetic Aperture Radar (POLSAR) systems describe the interactions between the electromagnetic wave and the target area by means of the Sinclair model. In a particular frequency band, the wave-media interactions over distributed areas are generally studied using the polarimetric covariance matrix (called also coherency when vectorizing in the Pauli basis).

The recently launched POLSAR systems are now capable of producing high quality images of the Earth's surface with meter resolution. The decrease of the resolution cell offers the opportunity to observe much thinner spatial features than the decametric resolution of the up-to-now available SAR images. However, higher scene heterogeneity leads

Thanks to Centre National d'Etudes Spatiales (CNES) for funding. to non-Gaussian polarimetric clutter modelling as the POLSAR data can no more be considered locally Gaussian, especially for urban regions. One commonly used fully polarimetric non-Gaussian clutter model is the product model [1]. For this model, the speckle presents a dual nature depending on the involved polarimetric descriptor:

- intensity: speckle can be considered as a nuisance parameter as the Gaussian kernel induces undesired spatial variations over homogeneous textureless areas,

- covariance matrix: speckle represents the useful signal as the covariance matrix is computed using the Gaussian kernel (texture appears as nuisance).

The objective of this paper is to present a new normalized coherency estimation technique based on the Spherically Invariant Random Vectors model [2]. The remainder of this paper is organized as follows. Sect. 2 is dedicated to the presentation of the proposed estimation scheme. The heterogeneity of polarimetric textured scenes is taken into account by coupling the ML normalized coherency estimator with adaptive neighborhoods driven on the scalar ML span estimators. In Sect. 3, the results obtained using the proposed approach are presented and compared to those given by the Gaussian ML estimator computed within boxcar neighborhoods. The use of the normalized coherency and the span as two separate descriptors of POLSAR data sets is also discussed.

\section{HETEROGENEOUS MODEL FOR POLARIMETRIC TEXTURED SCENES}

For POLSAR data, the Spherically Invariant Random Vectors (SIRV) model is a class of non-homogeneous Gaussian processes with random power: its randomness is induced by variations in the radar backscattering over different polarization channels. 


\subsection{Clutter modelling by SIRV processes}

In this paper, the polarimetric descriptors used are the target vectors in the Pauli basis $\vec{k}=\left[k_{1}, k_{2}, k_{3}\right]^{T}$ (monostatic acquisition). Consequently, the complex three dimensional measurement $\vec{k}$ is defined as the product between the independent complex Gaussian vector $\vec{z}$ (speckle), with zero mean and covariance matrix $[M]=E\left\{\vec{z} \vec{z}^{\dagger}\right\}$, and the square root of the positive random variable $\tau$ (texture):

$$
\vec{k}=\sqrt{\tau} \vec{z}
$$

where $\dagger$ denotes the conjugate transpose operator and $E\{\ldots\}$ the mathematical expectation.

When using the product model, an identification problem can be observed: the SIRV model is uniquely defined with respect to the covariance matrix parameter up to a multiplicative constant. For solving this identification problem, the covariance matrix has to be normalized. In the following the covariance matrix $[M]$ is normalized such that $\operatorname{Tr}\{[M]\}=m$, where $\operatorname{Tr}\{[M]\}$ denotes the trace of the matrix $[M]$ and $m$ is the dimension of the target vector ( $m=3$ for monostatic POLSAR acquisitions).

In Eq. 1, the normalized covariance matrix is an unknown parameter which can be estimated from Maximum Likelihood (ML) theory. In [3], Gini et al. derived the ML estimate $[\widehat{M}]$ of the normalized covariance matrix for deterministic texture:

$$
[\widehat{M}]=f([\widehat{M}])=\frac{m}{N} \sum_{i=1}^{N} \frac{\vec{k}_{i} \vec{k}_{i}^{\dagger}}{\vec{k}_{i}^{\dagger}\left[\widehat{M}^{-1} \vec{k}_{i}\right.},
$$

where $N$ is the number of available observations of the target vector. This approach has been used in [4] by Conte et al. to derive a recursive algorithm for estimating the matrix $[M]$. This algorithm consists of computing the Fixed Point of $f$ using the sequence $\left([M]_{i}\right)_{i \geq 0}$ defined by $[M]_{i+1}=f\left([M]_{i}\right)$. It has been shown in [3] and [4] that the estimation scheme from Eq. 2, developed under the deterministic texture case, yields also an approximate ML estimator under stochastic texture hypothesis. This study has been completed by the work of Pascal et al. [2], which recently established the existence and the uniqueness of the Fixed Point estimator of the normalized covariance matrix, as well as the convergence of the recursive algorithm whatever the initialization.

We propose to apply these results in estimating normalized coherence matrices for high resolution POLSAR data. The main advantage of this approach is that the local scene heterogeneity can be taken into account without any "a priori" hypothesis regarding the texture random variable $\tau$ (Eq. 2 does not depend on $\tau$ ). The obtained Fixed Point is the approximate ML estimate under the stochastic $\tau$ assumption and the exact ML under deterministic $\tau$ assumptions. Moreover, the normalized polarimetric coherency matrix estimated using the Fixed Point method is unbiased [2].
One important consequence of the imposed normalization condition is that the resulting normalized polarimetric coherency matrix (NC) does not fully describe the POLSAR data set any more. The NC descriptor reveals information concerning the polarimetric diversity only, the power information being lost. Nevertheless, the complete description of the original data set can be achieved by coupling $\mathrm{NC}$ with the span (or total power) descriptor $P$. By estimating the normalized coherency as the Fixed Point solution of Eq. 2, the derived NC is independent of the total power and it contains polarimetric information only. Using this matrix, it is possible to compute the SIRV span ML estimator for unknown deterministic $\tau$ as:

$$
\widehat{P}_{P W F}=\vec{k}^{\dagger}[\widehat{M}]^{-1} \vec{k}
$$

When compared to the conventional span estimator, the main advantage of the Polarimetric Whitening Filter (PWF) is that it takes into account the correlation between the different polarization channels in the whitening process [5].

Note also that the PWF span estimator from Eq. 3 can be extended, under iid and Gaussian hypotheses, yielding the Multilook Polarimetric Whitening Filter (MPWF):

$$
\widehat{P}_{M P W F}=\frac{1}{N} \sum_{i=1}^{N}{\overrightarrow{k_{i}}}^{\dagger}\left[\widehat{M}_{i}\right]^{-1}{\overrightarrow{k_{i}}}
$$

When the covariance matrix is estimated using the Sample Covariance matrix Normalized (SCN) estimator, the MPWF span from Eq. 4 is also the ML estimator of the texture for the Gaussian case and it is unbiased [5].

\subsection{Spatial support}

In the estimation process a certain number of samples must be gathered for deriving the observation vector. In this purpose, the boxcar sliding neighborhood (BN) is usually employed. The main inconvenient of $\mathrm{BN}$ non-adaptive neighborhood is that the available number of samples is directly proportional with the loss of spatial resolution. Experiments on real data sets have shown that the Intensity Driven Adaptive Neighborhood (IDAN) represents, on the whole, a good trade-off between preserving signal characteristics and gathering significant number of samples for parameter estimation [6].

However, recent studies have revealed that the original IDAN algorithm tends to introduce bias with respect to the radiometry information [7]. The main reasons consist in the use of a symmetric confidence interval around the mean for the Gamma distributed intensity and the estimation of the initial seed by the median computed within a $3 \times 3$ neighborhood. We propose to deal with this specific problems by operating several modifications on the original IDAN method. The resulting SDAN procedure (Span Driven Adaptive Neighborhood) is presented in Algorithm 1 and allows to use heterogeneous scene models, such as SIRV, in the estimation step. 
Another physical parameter which needs to be estimated is the total power. For the SIRV model, the PWF span estimator is the ML estimator, hence it should be applied for textured areas. However, on Gaussian textureless areas, a stronger speckle reduction can be obtain using the MPWF estimator. In practical applications, the PWF and the MPWF estimators should be applied as follows: on "Gaussian stationary" regions the best span estimator is the MPWF, while on "SIRV homogeneous" areas only, the PWF should be applied. We propose to deal with this trade-off by applying the LLMMSE criterion for the span estimation [8].

\section{RESULTS AND DISCUSSION}

As, for real data, it is hard to find reference regions with known coherency matrix, the effectiveness of the estimation schemes is demonstrated using simulated POLSAR data. The present paper proposes the analysis of two such estimation techniques: the Sample Covariance matrix Normalized coupled with the $7 \times 7$ Boxcar Neighborhood (BN-SCN) and the Fixed Point estimator coupled with the SDAN adaptive neighborhood (SDAN-FP). In all cases, the corresponding span image is estimated using the LLMMSE estimator.

The first POLSAR data set proposes four adjacent nonGaussian regions as presented in Fig. 1. Each of the four quadrants has associated a $\mathcal{K}$-distributed texture value [Fig. 1(a)] and a known theoretical covariance matrix. The texture coefficient of variation (CV) used for simulation equals 3 , which corresponds to a highly non-Gaussian clutter (urban areas). Fig. 1-(b) shows the corresponding amplitude color composition of the three target vector components.

The effectiveness of the Fixed Point estimator in compound Gaussian clutter can be observed in Fig. 1-(c),(d). While the BN-SCN normalized coherency [Fig. 1-(c)] presents a "patchy" appearance, the SDAN-FP estimation [Fig. 1-(d)] provides better visual homogeneity within each quadrant.

To illustrate the improvements in the conventional POLSAR processing chain, results obtained with high-resolution airborne data are reported. The POLSAR data set [Fig. 2-(a)] was acquired in October 2006 by the E-SAR system over the upper part of the Tacul glacier from the "Chamonix - Mont Blanc" test site, France. It represents a fully polarimetric (monostatic mode) L-band acquisition with a spatial resolution of approximately $1.5 \mathrm{~m}$ in range and azimuth. Visual assessment is carried out also with normalized coherency $[M]$ estimates. Color compositions, constructed from the diagonal elements of $[M]$ are computed for the two estimation techniques [Fig. 3-(b),(c)].

One key issue to be discussed is whether the normalized coherency matrix (NC) and the span should be aggregated in the final estimation step or not [Fig. 2-(d)]. Due to the SIRV model identification problem discussed in Sect. 2, the complete description of the POLSAR data set is achieved by estimating the span [Fig. 2-(b)] and the normalized coherency

\section{Algorithm 1 : Span-Driven-Adaptive-Neighborhood}

1. Minimal neighborhood $A N_{(i, j)}^{(I)}$ : in each pixel $(i, j)$ the minimal $A N_{(i, j)}^{(I)}$ is defined as the $3 \times 3 B N$.

(a) Seed normalized coherency matrix ML estimation using the Fixed Point estimator: $\left[M_{F P}^{(I)}\right](i, j)$.

(b) Seed span MPWF estimation: $p^{(I)}(i, j)$.

2. Region growing $A N_{(i, j)}^{(I I)}$ : the eight direct neighbors $\left(i^{\prime}, j^{\prime}\right)$ of the seed are accepted inside the $A N_{(i, j)}^{(I I)}$ provided that:

$$
1-C V \leq \frac{\vec{k}^{\dagger}\left(i^{\prime}, j^{\prime}\right) \cdot\left[M_{F P}^{(I)}\right]^{-1}(i, j) \cdot \vec{k}\left(i^{\prime}, j^{\prime}\right)}{p^{(I)}(i, j)} \leq 1+C V .
$$

The same procedure is applied for all the neighbors of the newly included pixels and so on. The region growing is iterated until either the number of pixels already included in the $A N_{(i, j)}^{(I I)}$ exceeds a predefined upper limit $N_{\max }$ or none of the new neighbors fulfills the test condition. The pixels which have already been tested but not accepted inside the $A N_{(i, j)}^{(I I)}$ are stored in a separate list (background pixels).

(a) Refined estimation of the seed normalized coherency matrix: a more reliable estimate of the normalized coherency $\left[M_{F P}^{(I I)}\right](i, j)$ is obtained using the pixels included in $A N_{(i, j)}^{(I I)}$.

3. Reinspection of the background pixels $A N_{(i, j)}$ : the background pixels $\left(i^{\prime \prime}, j^{\prime \prime}\right)$ of the list created in step 2 are tested again and aggregated in the $A N_{(i, j)}$ provided that:

$$
\begin{aligned}
& 1-T_{\text {low }} \cdot C V \leq \frac{\vec{k}^{\dagger}\left(i^{\prime \prime}, j^{\prime \prime}\right) \cdot\left[M_{F P}^{(I I)}\right]^{-1}(i, j) \cdot \vec{k}\left(i^{\prime \prime}, j^{\prime \prime}\right)}{p^{(I)}(i, j)} \\
& \frac{\vec{k}^{\dagger}\left(i^{\prime \prime}, j^{\prime \prime}\right) \cdot\left[M_{F P}^{(I I)}\right]^{-1}(i, j) \cdot \vec{k}\left(i^{\prime \prime}, j^{\prime \prime}\right)}{p^{(I)}(i, j)} \leq 1+T_{\text {high }} \cdot C V
\end{aligned}
$$

where the constants $T_{\text {low }}$ and $T_{\text {high }}$ are set in order to retain over per $=99 \%$ of the Gamma pdf.

Example: for $L_{e q}=3$, with $T_{\text {low }}=1.66$ and $T_{\text {high }}=5$, the AN retains per $=99.9 \%$ of the Gamma pdf.

[Fig. 2-(c)] independently. The NC describes the polarimetric diversity, while the span indicates the total received power. Moreover, the Fixed Point estimation of the normalized coherence does not depend on the span information. Given these facts, the joint analysis of the span and the normalized coherency presents several advantages with respect to the coherency matrix descriptor: separation between the total received power and the polarimetric information and estimation of the $\mathrm{NC}$ independently of the span. However, the span-NC description of POLSAR images raises new problems which still remain under investigation such as the use of span for testing the "matrix stationarity" condition for the normalized coherency estimation, or different clustering strategies better suited to capture the spatial distribution of different polarimetric signatures. 


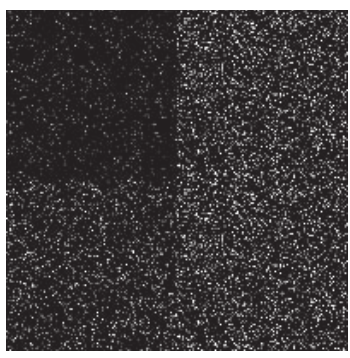

(a)

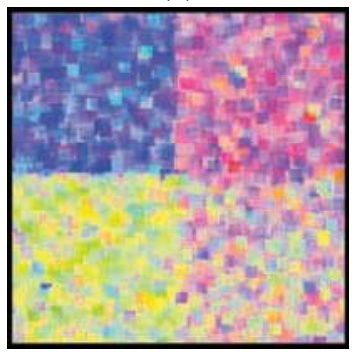

(c)

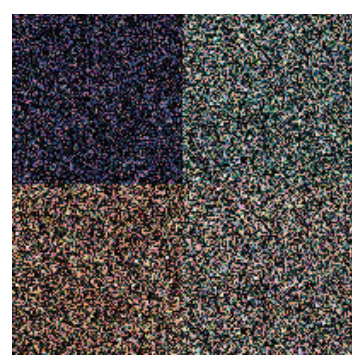

(b)

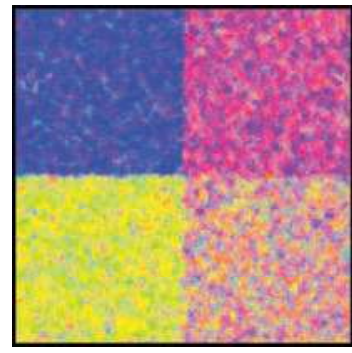

(d)
Fig. 1. Simulated POLSAR data, SIRV case $(200 \times 200$ pixels $)$ : (a) texture image and (b) amplitude color composition of the target vector elements $k_{1}-k_{3}-k_{2}$. Color composition of the normalized coherency diagonal elements $[M]_{11}-[M]_{33}-[M]_{22}$ estimated by: (c) BN-SCN and (d) SDAN-FP.

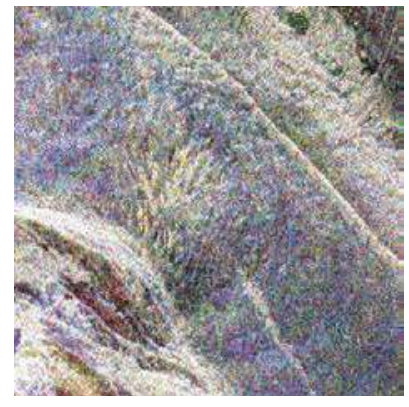

(a)

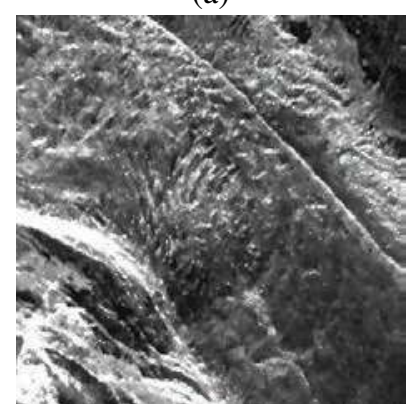

(b)

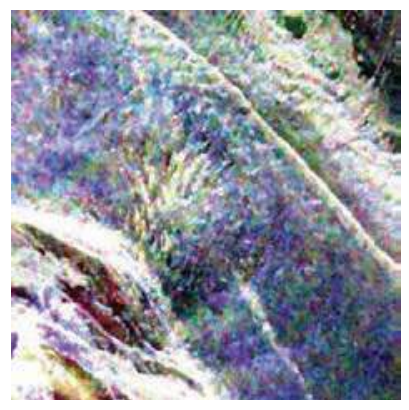

(d)

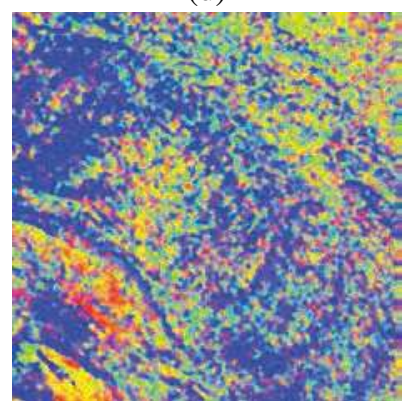

(c)
Fig. 2. E-SAR data $(400 \times 400$ pixels $)$ : (a) target vector elements $k_{1}-k_{3}-k_{2}$, (b) LLMMSE span estimated by SDAN-FP, (c) SDAN-FP normalized coherency diagonal elements $[M]_{11}-[M]_{33}-[M]_{22}$, (d) SDAN-FP coherency diagonal elements $[T]_{11-}-[T]_{33-}-[T]_{22}$ obtained as the product $[M] \cdot P \cdot m^{-1}$.

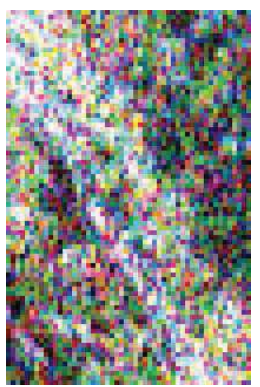

(a)

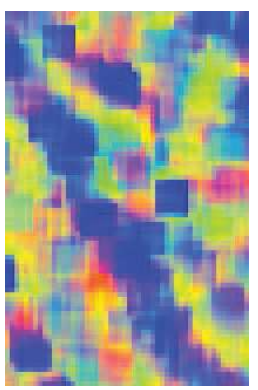

(b)

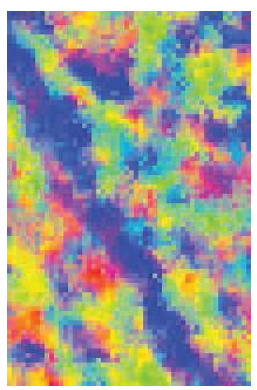

(c)
Fig. 3. E-SAR data (zoom 4x): (a) target vector elements $k_{1}-k_{3}-k_{2}$. Color composition of the normalized coherency diagonal elements $[M]_{11}-[M]_{33}-[M]_{22}$ estimated by: (b) BN-SCN and (c) SDAN-FP.

\section{CONCLUSION}

This paper presented a new estimation scheme for deriving normalized coherency matrices with high resolution POLSAR images. The proposed approach couples nonlinear ML estimators with span driven adaptive neighborhoods for taking the local scene heterogeneity into account.

\section{Acknowledgment}

The authors would like to thank the German Aerospace Center (DLR-HR) for providing the high resolution POLSAR images.

\section{REFERENCES}

[1] F. T. Ulaby, F. Kouyate, B. Brisco, and T. H. L. Williams, "Textural information in SAR images," IEEE Transactions on Geoscience and Remote Sensing, vol. GE-24, no. 2, pp. 235-245, 1986.

[2] F. Pascal, Y. Chitour, J. P. Ovarlez, P. Forster, and P. Larzabal, "Covariance structure maximum-likelihood estimates in compound gaussian noise: existence and algorithm analysis," IEEE Transactions on Signal Processing, vol. 56, no. 1, pp. 34-48, 2008.

[3] F. Gini and M. V. Greco, "Covariance matrix estimation for CFAR detection in correlated heavy tailed clutter," Signal Processing, vol. 82, no. 12, pp. 1847-1859, 2002.

[4] E. Conte, A. DeMaio, and G. Ricci, "Recursive estimation of the covariance matrix of a compound-Gaussian process and its application to adaptive CFAR detection," IEEE Transactions on Image Processing, vol. 50, no. 8, pp. 1908-1915, 2002.

[5] A. Lopes and F. Sery, "Optimal speckle reduction for the product model in multilook polarimetric SAR imagery and the Wishart distribution," IEEE Transactions on Geoscience and Remote Sensing, vol. 35, no. 3, pp. 632-647, 1997.

[6] G. Vasile, E. Trouvé, J. S. Lee, and V. Buzuloiu, "Intensity-DrivenAdaptive-Neighborhood technique for polarimetric and interferometric SAR parameters estimation," IEEE Transactions on Geoscience and Remote Sensing, vol. 44, no. 5, pp. 1609-1621, 2006.

[7] G. Vasile, Imagerie radar à synthse d'ouverture interférométrique et polarimétrique. Application au suivi des glaciers alpins, Ph.D. thesis, Université de Savoie, France, 2007.

[8] J. S. Lee, M. R. Grunes, and G. DeGrandi, "Polarimetric SAR speckle filtering and its impact on terrain classification," IEEE Transactions on Geoscience and Remote Sensing, vol. 37, no. 5, pp. 2363-2373, 1999. 